\title{
Superconducting Density of States in B-Doped Diamond
}

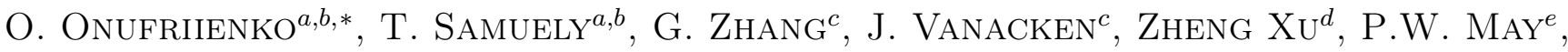 \\ P. SzaBÓ ${ }^{a, b}$, V.V. MoshChalKOV ${ }^{c}$ AND P. SAMUely ${ }^{a, b}$ \\ ${ }^{a}$ Centre of Low Temperature Physics, Institute of Experimental Physics, Slovak Academy of Sciences, \\ Watsonova 47, 04001 Košice, Slovakia \\ ${ }^{b}$ Faculty of Science, P.J. Šafárik University, 04001 Košice, Slovakia \\ ${ }^{c}$ INPAC-Institute for Nanoscale Physics and Chemistry, KU Leuven, \\ Celestijnenlaan 200D, B-3001 Heverlee, Belgium \\ ${ }^{d}$ School of Electrical, Computer and Energy Engineering, Arizona State University, Tempe, AZ 85281, USA \\ ${ }^{e}$ School of Chemistry, University of Bristol, Bristol BS8 1TS, UK
}

\begin{abstract}
In the presented work, we investigated the superconducting boron doped diamond polycrystalline film prepared by chemical vapor deposition by means of scanning tunneling microscopy/spectroscopy. Differential conductance spectra measured at various temperatures were used to obtain the values of superconducting critical temperature and energy gap. Comparing various theoretical models fitted to the differential conductance spectra measured at $0.5 \mathrm{~K}$ suggests weak pair breaking. However, this cannot account for the high $\frac{2 \Delta}{k_{\mathrm{B}} T_{\mathrm{C}}}$ ratio, which therefore indicates strong coupling.
\end{abstract}

DOI: 10.12693/APhysPolA.131.1033

PACS/topics: 74.70.Wz, 74.62.En, 74.55.+v

\section{Introduction}

Diamond is well-known as a perfect insulator. When doped with boron, however, it becomes a semiconductor [1]. What is more, in the beginning of the 21st century, the existence of superconductivity in boron doped diamond was discovered in samples grown by either high temperature high pressure method (HTHP) [2] or in thin films prepared by chemical vapor deposition (CVD) [3]. The transition temperature to the superconducting state was several times higher as in other boron-doped semiconductors, for example silicon [4], silicon carbide [5] and germanium [6]. The origin of the superconductivity in boron doped diamond remains unexplained, thus far, while it is clear that disorder plays a major role [7]. These discoveries boosted the study of boron-doped diamonds by scientists around the world significantly.

\section{Experiment}

The sample we studied was prepared by hot filament CVD method. The substrate, $\mathrm{SiO}_{2} / \mathrm{Si}$ seeded with diamond particles (15-25 nm diameter size) held at $800^{\circ} \mathrm{C}$, was located in a CVD reactor with $0.6 \% \mathrm{CH}_{4}$ in $\mathrm{H}_{2}$ gas mixture, thermally dissociated at $2200^{\circ} \mathrm{C}$. Boron doping was obtained by adding diborane $\left(\mathrm{B}_{2} \mathrm{H}_{6}\right)$ to the gas mixture with a $\mathrm{B}_{2} \mathrm{H}_{6} / \mathrm{CH}_{4}$ ratio of $2 \%$. The final product of boron-doped microcrystalline diamond (MCD) film had a thickness of $\approx 900 \mathrm{~nm}$, a mean grain size of $\approx 800 \mathrm{~nm}$ and the boron concentration of $\approx 1.5 \times 10^{21} \mathrm{~cm}^{-3}[8]$.

\footnotetext{
* corresponding author; e-mail:

oleksandr.onufriienko@student.upjs.sk
}

Our scanning tunneling microscopy and spectroscopy (STM/S) measurements were performed by means of a homemade low temperature STM head in Košice developed in collaboration with UAM Madrid [9], inserted in a commercial Janis SSV cryomagnetic system with ${ }^{3} \mathrm{He}$ refrigerator and controlled by Nanotec's Dulcinea SPM electronics. The tip was made of Au wire (99.99\% purity) and treated in situ by controlled collision with a clean $\mathrm{Au}$ surface [10]. A bias voltage of $5 \mathrm{mV}$ was applied to the tip. During STM, the tunneling current was kept constant at $1 \mathrm{nA}$ using a feedback loop.

In Fig. 1 we present the topography of the sample. The maximal surface corrugation was in the range $15-20 \mathrm{~nm}$, which is a considerably low value for a polycrystalline sample.

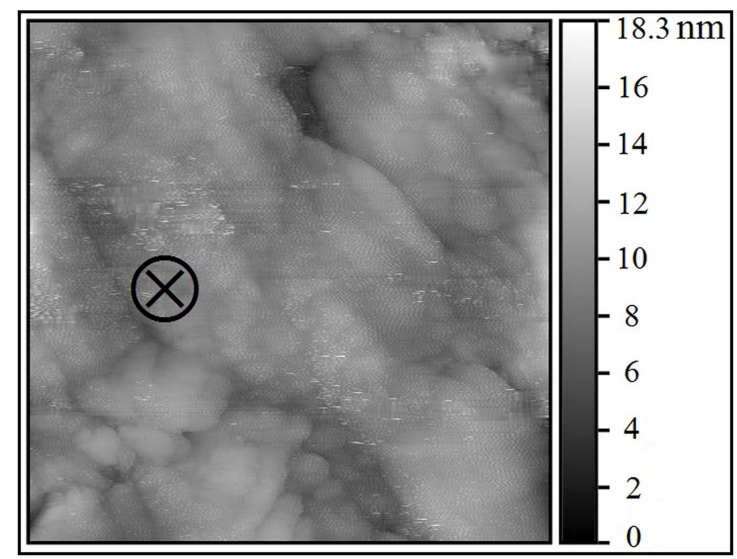

Fig. 1. The topography of the $500 \mathrm{~nm} \times 500 \mathrm{~nm}$ surface of the MCD film measured at the temperature of $500 \mathrm{mK}$. 
Observation of superconductivity was performed by measuring the tunneling $I-V$ characteristics at temperature range $0.5-8 \mathrm{~K}$. Subsequently, by numerical differentiation, the differential conductance vs. voltage spectra were obtained (Fig. 2). Because our metal tip has a flat density of states near the Fermi energy, the obtained spectra correspond to the thermally smeared local superconducting density of states (SDOS) of the sample. As can be seen in Fig. 2, the superconducting gap in the density of states is gradually suppressed with increase of temperature and the estimated superconducting critical temperature $T_{c}=7 \pm 0.5 \mathrm{~K}$. Such measurements were performed at numerous locations across the sample surface, yielding identical results within experimental error. We did not observe any spatial variation of SDOS as reported in previous studies of polycrystalline boron doped diamond [11-14]. Notably, the value of the differential conductance inside the superconducting gap is equal to zero (see Fig. 3). This is in steep contrast with previous studies on polycrystalline samples [11-14]. To our knowledge, such homogeneous hard gap was observed only in the case of a single crystal [15].

\section{Theoretical models}

For precise determination of the energy gap size we used various theoretical models. The first was the general theory that describes the SDOS, the BCS theory [16] for weakly coupled $s$-wave superconductors.

The tunneling spectra have been described by a theoretical expression for tunneling conductance

$$
\begin{aligned}
G_{n s}=\frac{\mathrm{d} I_{n s}}{\mathrm{~d} V} & = \\
G_{n n} & \int_{-\infty}^{\infty} \frac{\rho_{s}(E)}{\rho_{0}(0)}\left[-\frac{\partial f(E+e V)}{\partial(e V)}\right] \mathrm{d} E,
\end{aligned}
$$

where $G_{n s}$ is the superconducting conductance, $\rho_{s}$ is the SDOS that is defined as:

$$
\rho_{s}(E)=\rho_{0}(0) \operatorname{Re}\left(E / \sqrt{E^{2}-\Delta^{2}}\right),
$$

where $\rho_{0}$ is density of normal states, $\Delta$ is the superconducting energy gap.

In order to account for arbitrary smearing of the SDOS, Dynes modified the BCS SDOS by introducing an empirical complex parameter $\Gamma$ [17]:

$$
\rho_{s}(E, \Gamma)=\rho_{0}(0) \operatorname{Re}\left(\frac{E-\mathrm{i} \Gamma}{{\sqrt{(E-\mathrm{i} \Gamma)^{2}}-\Delta^{2}}^{2}}\right) .
$$

Another model that we employed, results from the Abrikosov-Gor'kov theory and accounts for magnetic impurities [18, 19].

The so called Maki model employs the pair braking parameter $\alpha$. The density of states is given by

$$
\rho_{s}(E)=\rho_{0}(0) \operatorname{Re}\left(u / \sqrt{u^{2}-1}\right) .
$$

The variable $u$ is defined by the nonlinear equation:

$$
u=E / \Delta+u / \sqrt{1-u^{2}} \text {. }
$$

To fit the model to our experimental data, we used the analytical solution of Eq. (5) given in the Appendix of [20].

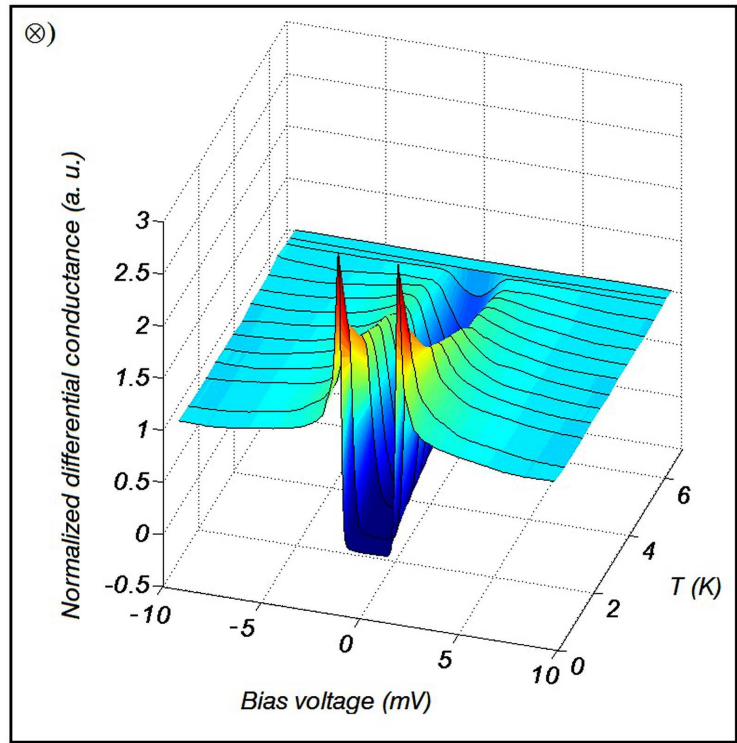

Fig. 2. Temperature dependences of the differential conductance in the position denoted by $\otimes$ in Fig. 1 .

\section{Discussion}

All three above mentioned models were fitted to the experimental data, as depicted in Fig. 3.

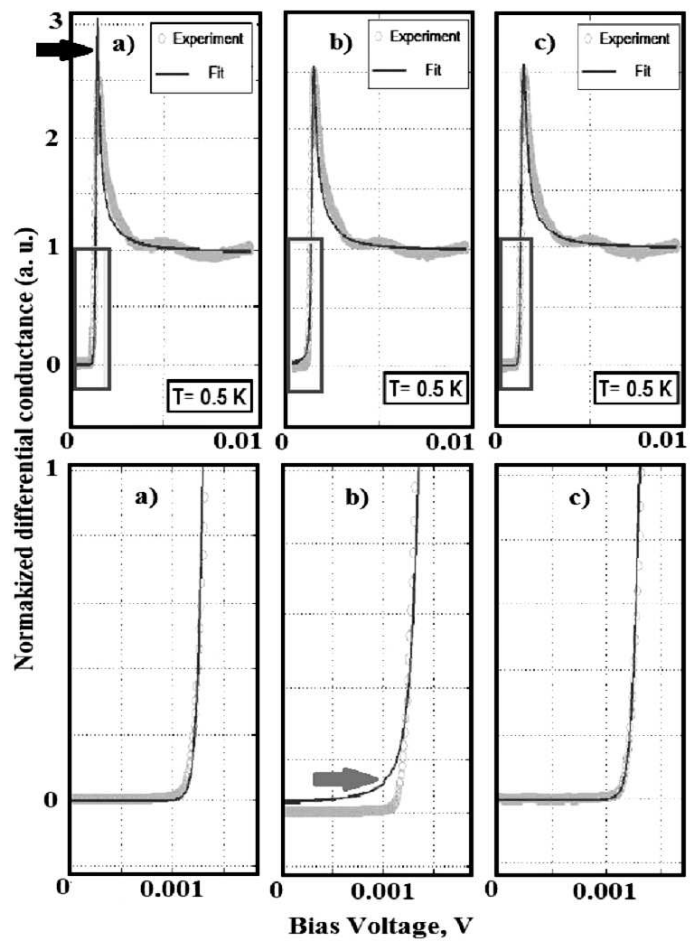

Fig. 3. Differential conductance at $T=500 \mathrm{mK}$ obtained by experiment (grey circles), and from theoretical models (black lines) with $\Delta=1.44 \mathrm{meV}$ : (a) BCS model, (b) Dynes model with $\Gamma=0.02 \mathrm{meV}$ and (c) Maki model with $\alpha=0.01$. 
All three models used $T=0.5 \mathrm{~K}$ and yielded $\Delta=$ $1.44 \mathrm{meV}$ in the fitting process. In the case of a simple BCS model (Fig. 3a), the coherence peak (indicated by the black arrow) clearly overshoots the experimental data. The suppressed coherence peak can be modeled using the Dynes modification of the spectrum, plotted in Fig. 3b. By fitting, the value of $\Gamma=0.02 \mathrm{meV}$ was obtained. In this case, however, the differential conductance values inside the superconducting gap deviate from the experimental ones, which are equal to zero, as indicated by the grey arrow. Finally, we fitted the third model (Fig. 3c). With the pair breaking parameter $\alpha=0.01$, we achieved both, the suppression of the coherence peaks and differential conductance values inside the superconducting gap equal to zero.

\section{Conclusions}

Using the low temperature scanning tunneling spectroscopy we determined the critical temperature $T_{c}=$ $7 \pm 0.5 \mathrm{~K}$ of the superconducting boron doped diamond polycrystalline film prepared by chemical vapor deposition. Also, by fitting the spectra, we obtained the value of the superconducting gap $\Delta=1.44 \mathrm{meV}$. Hence, we obtained the ratio $\frac{2 \Delta}{k_{\mathrm{B}} T_{\mathrm{C}}} \approx 4.8$, which is higher than the standard BCS value of 3.52. Moreover, by fitting different theoretical models to the spectrum acquired at the lowest temperature, we established that the experimental data is best interpreted by using a theoretical model that involves pair breaking. Nevertheless, the parameter value $\alpha=0.01$ indicates weak pair breaking. Therefore, the large gap to $T_{c}$ ratio cannot be explained by pair breaking and probably stems from strong coupling. The origin of the strong coupling, as well as the pair breaking, remains for further investigations.

\section{Acknowledgments}

This work was supported by the ERDF EU (European Union European regional development fond) grant, under the contract No. ITMS 26220120005, APVV 0605-14 and VEGA 1-0409-15, VEGA 2-0149-16.

\section{References}

[1] A.W.C. Williams, E.C. Lightowlers, A.T. Collins, J. Phys. C 3, 1727 (1970).

[2] E.A. Ekimov, V.A. Sidorov, E.D. Bauer, N.N. Mel'nik, N.J. Curro, J.D. Thompson, S.M. Stishov, Nature 428, 542 (2004).

[3] Y. Takano, M. Nagao, I. Sakaguchi, M. Tachiki, T. Hatano, K. Kobayashi, H. Umezawa, H. Kawarada, Appl. Phys. Lett. 85, 2851 (2004).
[4] E. Bustarret, C. Marcenat, P. Achatz, J. Kaĉmarĉik, F. Lévy, A. Huxley, L. Ortéga, E. Bourgeois, X. Blas, D. Débarre, J. Boulmer, Nature (London) 444, 465 (2006).

[5] Z.A. Ren, J. Kato, T. Muranaka, J. Akimitsu, M. Kriener, Y. Maeno, J. Phys. Soc. Jpn. 76, 103710 (2007).

[6] T. Herrmannsdörfer, V. Heera, O. Ignatchik, M. Uhlarz, A. Mücklich, M. Posselt, H. Reuther, B. Schmidt, K.H. Heinig, W. Skorupa, M. Voelskow, C. Wündisch, R. Skrotzki, M. Helm, J. Wosnitza, Phys. Rev. Lett. 102, 217003 (2009).

[7] E. Bustarret, J. Kačmarčik, C. Marcenat, E. Gheeraert, C. Cytermann, J. Marcus, T. Klein, Phys. Rev. Lett. 93, 237005 (2004).

[8] P.W. May, W.J. Ludlow, M. Hannaway, P.J. Heard, J.A. Smith, K.N. Rosser, Diamond Relat. Mater. 17, 105 (2008).

[9] J.G. Rodrigo, H. Suderow, S. Vieira, E. Bascones, F. Guinea, J. Phys. Condens. Matter 16, R1151 (2004).

[10] T. Samuely, P. Szabó, V. Komanický, J.G. Rodrigo, S. Vieira, P. Samuely, Acta Phys. Pol. A 118, 1038 (2010).

[11] G. Zhang, S. Turner, E.A. Ekimov, J. Vanacken, M. Timmermans, T. Samuely, V.A. Sidorov, S.M. Stishov, Y. Lu, B. Deloof, B. Goderis, G. Van Tendeloo, J. Van de Vondel, V.V. Moshchalkov, Adv. Mater. 26, 2034 (2014).

[12] B.L. Willems, V.H. Dao, J. Vanacken, L.F. Chibotaru, V.V. Moshchalkov, I. Guillamón, H. Suderow, S. Vieira, S.D. Janssens, O.A. Williams, K. Haenen, P. Wagner, Phys. Rev. B 80, 224518 (2009).

[13] F. Dahlem, P. Achatz, O.A. Williams, D. Araujo, E. Bustarret, H. Courtois, Phys. Rev. B 82, 033306 (2010).

[14] T. Nishizaki, Y. Takano, M. Nagao, T. Takenouchi, H. Kawarada, N. Kobayashi, J. Phys. Chem. Solids 69, 3027 (2008).

[15] B. Sacépé, C. Chapelier, C. Marcenat, J. Kačmarčik, T. Klein, M. Bernard, E. Bustarret, Phys. Rev. Lett. 96, 097006 (2006).

[16] J. Bardeen, L.N. Cooper, J.R. Schrieffer, Phys. Rev. 108, 1175 (1957).

[17] R.C. Dynes, V. Narayanamurti, J.P. Garno, Phys. Rev. Lett. 41, 1509 (1978).

[18] R.D. Parks, Superconductivity, Vol. 1 and 2, Marcel Dekker, New York 1969, p. 1035.

[19] A.A. Abrikosov, L.P. Gor'kov, Sov. Phys. JETP 12, 1243 (1961).

[20] M. Assig, M. Etzkorn, A. Enders, W. Stiepany, C.R. Ast, K. Kern, Rev. Sci. Instrum. 84, 033903 (2013). 